\title{
Gum arabic-coated radioactive gold nanoparticles cause no short-term local or systemic toxicity in the clinically relevant canine model of prostate cancer
}

\author{
This article was published in the following Dove Press journal: \\ International Journal of Nanomedicine \\ 28 October 2014 \\ Number of times this article has been viewed
}

\author{
Sandra M Axiak-Bechtel' \\ Anandhi Upendran ${ }^{2,3}$ \\ Jimmy C Lattimer' \\ James Kelsey ${ }^{3,4}$ \\ Cathy S Cutler ${ }^{4}$ \\ Kim A Selting' \\ Jeffrey N Bryan' \\ Carolyn J Henry ${ }^{1,5}$ \\ Evan Boote ${ }^{6}$ \\ Deborah J Tate' \\ Margaret E Bryan ${ }^{7}$ \\ Kattesh V Katti ${ }^{3,8}$ \\ Raghuraman Kannan ${ }^{3,8}$ \\ 'Department of Veterinary Medicine \\ and Surgery, ${ }^{2}$ Department of Physics, \\ University of Missouri, Columbia, \\ MO, USA; ${ }^{3}$ Nanoparticle Biochem, \\ Inc., and Shasun-NBI LLC, Columbia, \\ MO, USA; ${ }^{4}$ Missouri University \\ Research Reactor, ${ }^{5}$ Department \\ of Internal Medicine, University \\ of Missouri, Columbia, MO, USA; \\ ${ }^{6}$ Spectrum Health, Grand Rapids, \\ MI, USA; ${ }^{7}$ Department of Statistics, \\ ${ }^{8}$ Department of Radiology, University \\ of Missouri, Columbia, MO, USA
}

\begin{abstract}
Introduction: Gum arabic-coated radioactive gold nanoparticles (GA- ${ }^{198} \mathrm{AuNPs}$ ) offer several advantages over traditional brachytherapy in the treatment of prostate cancer, including homogenous dose distribution and higher dose-rate irradiation. Our objective was to determine the short-term safety profile of GA- ${ }^{198}$ AuNPs injected intralesionally. We proposed that a single treatment of GA- ${ }^{198} \mathrm{AuNPs}$ would be safe with minimal-to-no evidence of systemic or local toxicity.
\end{abstract}

Methods: Nine dogs with spontaneously occurring prostatic cancer were treated. Injections were performed with ultrasound or computerized tomography guidance. Complete blood counts, chemistry panels, and urinalyses were performed at weekly intervals for 1 month and imaging was repeated 4 weeks postinjection. Planar scintigraphic images were obtained within 30 minutes of injection.

Results: No statistically significant difference was found in any hematologic or biochemical parameter studied, nor was any evidence of tumor swelling or abscessation found in eight dogs with repeat imaging; one dog died secondary to urethral obstruction 12 days following injection. At 30 minutes postinjection, an average of 53\% of injected dose in seven dogs was retained in the prostate, with loss of remaining activity in the bladder and urethra; no systemic uptake was detected.

Conclusion: GA- ${ }^{198} \mathrm{AuNP}$ therapy had no short-term toxicity in the treatment of prostatic cancer. While therapeutic agent was found in the prostate immediately following injection, some loss of agent was detected in the bladder and urethra. Localization of radioactivity within the prostate was lower than anticipated and likely due to normal vestigial prostatic ducts. Therefore, further study of retention, dosimetry, long-term toxicity, and efficacy of this treatment is warranted prior to Phase I trials in men.

Keywords: brachytherapy, prostatic cancer, safety trial

\section{Introduction}

Prostate cancer (PC) is the most common cancer diagnosed in men, of which over 28,000 died from this disease in the United States in 2012. ${ }^{1}$ Current treatment modalities for local control of cancer include radical prostatectomy, external beam radiation therapy, and brachytherapy. Brachytherapy has the advantage of emitting short-range radiation directly to the tumor, thereby protecting normal tissues with decreased offtarget effects compared to external beam radiation therapy. ${ }^{2-4}$ However, off-target effects such as urethral stricture, rectal bleeding, and erectile dysfunction are still seen and, in part, may be related to the number of implants placed for appropriate
Correspondence: Sandra M Axiak-Bechtel University of Missouri, 900 East Campus Drive, Columbia, MO, 6521I, USA

$\mathrm{Tel}+$ I 573882782 I

Fax +I 5738840705

Email bechtels@missouri.edu 
dosing to the tumor. ${ }^{4,5}$ Furthermore, failure of treatment occurs in $25 \%$ or more of men with high Gleason score PC. This localized failure is due to multiple factors, including large tumor volume and difficulty achieving a geometrically optimal distribution of seeds, resulting in heterogeneous dose distribution increasing the rate of significant side effects. ${ }^{5-7}$ Since an increase in side effects can be expected with increased number of implants and higher radiation dose, a safe and homogenously distributed form of brachytherapy with reduced placement trauma would be ideal to improve disease control while limiting side effects.

Brachytherapy with low dose-rate radiation (LDR) (palladium-103 and iodine-125) and high dose-rate radiation (HDR) (gold-198 and iridium-192) has been used to treat PC. ${ }^{6-9}$ Recent studies have indicated that HDR may be more effective than LDR for high-grade PC. ${ }^{3,8}$ LDR modalities with longer half-lives give a lower initial dose rate to tumor, which can be less effective in treating fast-growing or low- $\alpha / \beta$-ratio tumors such as high-grade PC. ${ }^{4,8}$ In fact, HDR with ${ }^{192}$ Ir has been used successfully with optimal dose distribution, but with the disadvantage of multiple invasive procedures required for optimal therapy. ${ }^{3}$ Radioactive gold $\left({ }^{198} \mathrm{Au}\right)$ has an average gamma-ray energy of $412 \mathrm{keV}$; this isotope supplies a greater dose depth and less anisotropy in tissue with a shorter half-life (2.7 days) compared to lowerenergy radionuclides when used in shielded discrete sources. When used as a non-shielded source, the large majority of the dose is derived from the beta-particle component of the decay which has an average energy of approximately $320 \mathrm{keV} .{ }^{4}$ In men, implanted ${ }^{198} \mathrm{Au}$ seeds provided the benefits of HDR including greater dose depth and delivering prescribed dose in a shorter period of time with limited invasiveness (only one procedure for implantation compared to multiple treatments with ${ }^{192} \mathrm{Ir}$ ); however, ${ }^{198} \mathrm{Au}$ seeds still have the potential disadvantages of other seed brachytherapy treatments, including heterogeneous dose distribution. ${ }^{8}$

${ }^{198} \mathrm{Au}$ nanoparticles (NPs) have the potential to overcome the disadvantage of heterogeneous dose distribution with traditional seed brachytherapy due to their size. Gum arabic, a glycoprotein, is used as a stabilizer for ${ }^{198} \mathrm{AuNPs}$ and provides a nontoxic coating that prevents aggregation in vivo. ${ }^{10} \mathrm{NPs}$ of up to $100 \mathrm{~nm}$ tend to accumulate in tumors due to the irregularity of tumor vessels, large pore size, and poor lymphatic drainage. ${ }^{11}$ The hydrodynamic diameter of gum arabic-coated ${ }^{198} \mathrm{AuNP}$ (GA- ${ }^{198} \mathrm{AuNP}$ ) is $85 \mathrm{~nm}$ and may permit effective penetration of the particles into porous tumor vasculature and diffusion through the interstitium of the tumor. ${ }^{11,12}$ Evaluation of biodistribution of nonradioactive gum arabic-coated gold NP (GA-AuNP) in mice and pigs showed distribution to the liver and spleen when injected intravenously. ${ }^{13,14}$ Therefore, any NP not retained in the tumor and entering systemic circulation would likely localize in one of these organs following vascular clearance. In a PC3 implanted SCID mouse model study, intratumoral injection of the nonradioactive surrogate of GA- ${ }^{198} \mathrm{AuNPs}$ resulted in homogenous distribution throughout the tumor on computed tomography (CT) scan 24-hours postinjection. ${ }^{15}$ In another previously reported study using the prostate tumor mouse model, $408 \mu \mathrm{Ci}(30 \mu \mathrm{L})$ of GA- ${ }^{198} \mathrm{AuNPs}$ were administered as a single injection into the prostate tumor and compared to a control group. Greater than $75 \%$ of radioactivity was retained within the tumor at 24 hours, and treated mice had a tumor volume reduction of $82 \%$ compared to controls at the end of the 30-day study period. ${ }^{15,16}$ No off-target effects were noted in any treated mouse other than transient and reversible weight loss. ${ }^{16}$ The homogenous distribution revealed in the mouse model suggested further studies should utilize the homogenous distribution model for dosimetry. These studies together demonstrated safety, tolerability, uniform distribution, and efficacy of GA- ${ }^{198}$ AuNPs. Therefore, GA- ${ }^{198}$ AuNPs provide promise for an effective, minimally invasive treatment with fewer adverse effects than current standards of care. ${ }^{16}$ Furthermore, several gold NPs of different sizes and shapes have been reported in the literature. The prominent NPs include glutathione, bovine serum albumin, or polyethylene glycol-conjugated gold NPs. Even though these NPs have ideal in vivo characteristics, they are not easily synthesizable using ${ }^{198} \mathrm{Au}$ as a precursor. ${ }^{17-19}$ On the other hand, GA- ${ }^{198}$ AuNPs utilized in the present study were synthesized using a one-step procedure directly from ${ }^{198} \mathrm{Au}$. This is an important advantage for clinical utilization.

The objective of the current study was to determine the safety of GA- ${ }^{198}$ AuNPs injected intralesionally in the treatment of naturally occurring prostatic cancer, using companion dogs with spontaneously occurring PC as a translational model. We proposed that treatment would be safe, systemic toxicoses would not occur, and that short-term off-target effects would not be evident in this set of dogs.

\section{Materials and methods}

\section{Animals}

This project was approved by the University of Missouri Animal Care and Use Committee (ACUC protocol \#6488). Dogs with spontaneously occurring prostatic cancer were recruited from those routinely treated at the University of Missouri Veterinary Medical Teaching Hospital. Castrated 
or intact male dogs of any age, breed, or size diagnosed with prostatic carcinoma by cytology or biopsy, with a life expectancy of greater than 6 weeks and informed owner consent, were eligible for enrollment. Metastasis to lymph nodes in the sublumbar region was permitted, with the intent to treat affected nodes by a separate injection of GA- ${ }^{198}$ AuNPs if affected. Dogs were excluded if a significant comorbidity was present, concurrent bladder tumor or extension of prostate tumor into the bladder was present, or if metastasis to any site other than lymph nodes in the sublumbar region was identified and determined to result in a life expectancy of less than 6 weeks. Pretreatment evaluation was performed within 2 weeks of planned injection and included complete blood count, plasma biochemistry panel, urinalysis, thoracic radiographs, abdominal ultrasound, and abdominal CT scan. A urethral stent was placed in some dogs if a contrast cystourethrogram was performed and evidence of urethral obstruction was established.

Nine dogs were enrolled. All dogs were castrated and mixed breed was the most common breed of dog represented $(n=3)$. Other breeds included were Nova Scotia duck tolling retriever $(n=1)$, Boston terrier $(n=1)$, beagle $(n=1)$, chow chow $(n=1)$, Beauceron $(n=1)$, and Irish setter $(n=1)$. Ages ranged from 8 to 12 years and body weights from 6 to $43 \mathrm{~kg}$, with a mean of $24.5 \mathrm{~kg}$. Eight dogs had cytologic confirmation of carcinoma and one dog had histopathologic confirmation. Three dogs had prior chemotherapy (mitoxantrone or carboplatin) and nonsteroidal anti-inflammatory treatment, and four dogs had treatment with nonsteroidal anti-inflammatory drugs alone prior to trial enrollment; two dogs had no prior therapy. Two dogs had metastasis to lymph nodes in the sublumbar region at initial evaluation and one dog had a small soft tissue nodule in the lungs found on CT scan, potentially representing pulmonary metastasis. This latter dog's tumor had been refractory to prior chemotherapy; since this finding was incidental and not thought to be life limiting, this dog was enrolled in the study. Three dogs had urethral stents placed prior to GA- ${ }^{198} \mathrm{AuNP}$ injection following a contrast cystourethrogram that revealed significant urethral obstruction caused by tumor invasion. Tumor volume ranged from $10.8-69.4 \mathrm{~cm}^{3}$ and was variable based on dog size and rate of tumor growth (Table 1). In all dogs, normal prostate was not distinguishable from prostatic tumor. Lymph nodes were not included in tumor volume measurements.

\section{GA- ${ }^{198} A u N P$ production}

University of Missouri Research Reactor irradiation facilities were used for the production of ${ }^{198} \mathrm{Au}$ and the GA- ${ }^{198} \mathrm{AuNPs}$ in the volume and dose requested by previously published methods with slight modifications. ${ }^{10}$ Briefly, gold foil was irradiated at a flux of $8 \times 10^{13}$ neutrons $/ \mathrm{cm}^{2} / \mathrm{s}$; the radioactive foil was then dissolved with aquaregia, dried, and reconstituted in hydrochloric acid to yield $\mathrm{H}^{198} \mathrm{AuCl}_{4}$. GA- ${ }^{198} \mathrm{AuNPs}$ were produced by the addition of the reducing agent tris hydroxymethyl phosine-alanine $\left(\mathrm{P}\left(\mathrm{CH}_{2} \mathrm{NHCH}\left(\mathrm{CH}_{3}\right)-\mathrm{COOH}\right)_{3}\right.$ in the presence of gum arabic. The final solution was brought to a $\mathrm{pH}$ of 7 and sterile filtered. The gold concentration in the final nanoparticulate suspension was $0.325 \mathrm{mg} / \mathrm{mL}(1.6 \mathrm{mM}$; $\left.9.026 \times 10^{15} \mathrm{NPs}\right)$. Production of GA- ${ }^{198} \mathrm{AuNPs}$ was confirmed by quality control tests including radio thin layer chromatography and ultraviolet-visible spectroscopy. Radio thin layer chromatography confirmed that $>98 \%$ of the $\mathrm{H}^{198} \mathrm{AuCl}_{4}$ was in the nanoparticulate form. The physicochemical parameters were established using the nonradioactive analogue (GAAuNP). Particles had a hydrodynamic diameter of $85 \mathrm{~nm}$ $( \pm 20 \mathrm{~nm})$ and zeta potential of $24.5 \pm 1.5 \mathrm{mV}$ as determined by dynamic light scattering measurements. The core size of

Table I Dog weights, three-dimensional tumor volume measurements, percent volume change, and total activity injected into each prostate tumor

\begin{tabular}{lllllll}
\hline Dog & $\begin{array}{l}\text { BED group } \\
(\mathbf{G y})\end{array}$ & $\begin{array}{l}\text { Weight } \\
\mathbf{( k g )}\end{array}$ & $\begin{array}{l}\text { Pretreatment } \\
\text { tumor volume } \mathbf{( \mathbf { c m } ^ { 3 } )}\end{array}$ & $\begin{array}{l}\text { Posttreatment tumor } \\
\text { volume } \mathbf{( \mathbf { c m } ^ { 3 } )}\end{array}$ & $\begin{array}{l}\text { Volume } \\
\text { decrease } \mathbf{( \% )}\end{array}$ & $\begin{array}{l}\text { Activity injected } \\
(\mathbf{m C i})\end{array}$ \\
\hline 1 & 50 & 22.4 & 10.81 & 7.52 & 30 & 4.8 \\
2 & 50 & 11 & 20.06 & 20.29 & -1 & 3.0 \\
3 & 105 & 29.7 & 50.94 & Death prior to week 4 & N/A & 6.2 \\
4 & 105 & 18.8 & 27.81 & 31.36 & -12 & 4.5 \\
5 & 105 & 33.7 & 34.06 & 34.1 & -0.1 & 5.8 \\
6 & 105 & 39.6 & 69.4 & 71.71 & -3 & 13.8 \\
7 & 105 & 21.6 & 27.44 & 12.3 & 55 & 9.1 \\
8 & 105 & 6.6 & 20.47 & 19.85 & 3 & 6.4 \\
9 & 105 & 37 & 53.65 & 67.57 & -26 & 8.8 \\
\hline
\end{tabular}

Abbreviation: BED, biologically equivalent dose. 
the particles was $12-15 \mathrm{~nm}$ as determined by transmission electron microscopy. ${ }^{16}$

\section{Dosimetry}

Naturally-occurring canine prostate tumors differ in size and geometry in proportion to the breed size and duration of growth. Therefore, dosimetry was calculated separately for each dog using the published standard American Association of Physicists in Medicine Task Group-43 formulation with tumor volume calculated on pretreatment CT scans. ${ }^{4,20}$ All dogs were injected with a quantity of GA- ${ }^{198}$ AuNPs calculated to deliver a biologically equivalent dose (BED) of 50 Gy for feasibility $(n=2)$ or 105 Gy $(n=7)$ for treatment. The following formulas were used for calculation:

$$
\begin{aligned}
& \mathrm{T}_{\text {eff }}=1+(\beta / \alpha) \times\left[\mathrm{D}_{\mathrm{i}} /(\mu-\lambda)\right] \times\left[1 /\left(1-\mathrm{e}^{\left.-\lambda \mathrm{T}_{\text {eff }}\right)}\right.\right. \\
& \times\left[1-\mathrm{e}^{-2 \lambda \mathrm{T}_{\text {eff }}}-(2 \lambda / \mu+\lambda) \times\left(1-\mathrm{e}^{-(\mu+\lambda) \mathrm{T}_{\text {eff }}}\right]\right. \\
& \mathrm{D}\left(\mathrm{T}_{\text {eff }}\right)=\mathrm{D}_{\mathrm{i}}\left(1.44 \mathrm{~T}_{1 / 2}\right)\left(1-\mathrm{e}^{0.693 \mathrm{~T}_{\text {eff }} \mathrm{T}_{1 / 2}}\right) \\
& \mathrm{BED}=\mathrm{D}\left(\mathrm{T}_{\mathrm{eff}}\right) \operatorname{RE}\left(\mathrm{T}_{\mathrm{eff}}\right)-\ln 2\left(\mathrm{~T}_{\mathrm{eff}} / \alpha \mathrm{T}_{\mathrm{p}}\right) \\
& \mathrm{TCP}=\exp \left[-\mathrm{N}_{0} \mathrm{e}^{(-\alpha \mathrm{BED})}\right] \\
& \alpha=0.15 / \mathrm{Gy} \\
& \beta=0.05 / \mathrm{Gy}^{2}
\end{aligned}
$$

where $\mathrm{D}$ is dose in cGy; $\mathrm{D}_{\mathrm{i}}$ is initial dose rate; e is average beta particle energy in $\mathrm{MeV}, \sim 0.321$ for ${ }^{198} \mathrm{Au}$; exp is exponential function; $\lambda$ is decay constant for ${ }^{198} \mathrm{Au} ; \lambda_{\text {eff }}$ is effective decay constant $\left(0.693 / \mathrm{T}_{1 / 2 \mathrm{p}}\right) ; \mu$ is time constant for sublethal repair, inversely proportional to the repair half-time; $\mathrm{N}_{0}$ is number of tumor cells initial present; $\mathrm{Re}$ is repair effectiveness; $\mathrm{T}_{1 / 2}$ of ${ }^{198} \mathrm{Au}$ is 2.7 days; TCP is tumor control probability; $\mathrm{T}_{\mathrm{p}}$ is potential doubling time of prostate tumor cells (42 days); and $\mathrm{T}_{\text {eff }}$ is effective half-life in days.

These calculations yielded a dose of $0.17 \mathrm{mCi}{ }^{198} \mathrm{Au} / \mathrm{gram}$ of tissue to deliver a BED of 105 Gy assuming $100 \%$ retention and homogenous distribution of the NPs through the tissue of interest. Since retention was actually $75 \%$ in the mouse SCID PC3 model, a target injection dose was calculated to approximately $0.20 \mathrm{mCi} / \mathrm{g}$ of tumor tissue, with tumor volume determined using three-dimensional measurements obtained from pretreatment CT scans. The estimated dose based on these calculations was $63,630 \mathrm{rad} / \mathrm{mCi} / \mathrm{g}$ of prostate tumor tissue. Distribution was monitored immediately postinjection using nuclear scintigraphy as described below.

Intratumoral injection of GA- ${ }^{198} \mathrm{AuNPs}$ was performed using aseptic technique and ultrasound $(n=1)$ or CT-guided $(n=8)$ needle placement under general anesthesia. Needle placement varied based on tumor size and position within the pelvic canal, with planned injection sites $1 \mathrm{~cm}$ apart. The volume of the tumor and the number of required injection points were predetermined using a previously performed diagnostic CT scan. The dogs were positioned in dorsal recumbency and 25-gauge spinal needles were inserted into the prostate using ultrasound $(n=1)$ or CT $(n=8)$ guidance at approximately $1 \mathrm{~cm}$ intervals. The number of needles used varied from two to eight. Based on the volume determinations from previous imaging studies, the specific activity of the radioisotope in the suspension was adjusted to a volume of 100-200 $\mu \mathrm{L}$ at each injection site, with the total prescribed dose evenly divided among the total number of injection sites. Following recovery from anesthesia, dogs were housed in standardized radiation isolation facilities. Planar images were obtained by whole body scintigraphy within 30 minutes postinjection to monitor the presence of GA- ${ }^{198}$ AuNPs within the prostate. Region of interest (ROI) evaluation was used to estimate the amount of GA- ${ }^{198} \mathrm{AuNP}$ present in the prostate, urethra, and bladder. The prostate to bladder retention ratio of GA- ${ }^{198}$ AuNP was calculated for each dog imaged. All dogs were hospitalized in radiation isolation until acceptable radioactivity levels were reached for release criteria as determined by the Radiation Safety Officer. Appetite, attitude, urination, and defecation were monitored twice daily, and subjective pain score was monitored once daily while hospitalized. Intervention for pain included oral or injectable opioids at the discretion of the attending veterinarian. Nonsteroidal anti-inflammatory drugs were not allowed unless started at least 2 weeks prior to study. Monitoring included weekly complete blood count, serum chemistry panel, and urinalysis for 4 weeks following release from radiation isolation. Wilcoxon signed rank test was used to compare the following values pre- and posttreatment in all dogs: white blood cell count; red blood cell count; neutrophil count; platelet count; liver enzymes (alkaline phosphatase and alanine aminotransferase); and renal values (blood urea nitrogen and creatinine). A CT scan was obtained 4 weeks after treatment to identify evidence of posttreatment tumor swelling or abscessation. Prostatic volume at each time point was calculated in two different ways: using the standard geometric formula for calculating the volume of an ellipsoid; and the length $\times$ width $\times$ height measurements times pi divided by 6 method commonly used in oncology. Measurements were also obtained from a three-dimensional computerized system which calculates the volume of the prostate based on the cross-sectional area and voxel thickness for each section of the prostate. No chemotherapy or radiotherapy other than GA- ${ }^{198} \mathrm{AuNP}$ was allowed during the 4 -week trial period. 


\section{Results}

Two dogs were treated with a single treatment to a BED of $50 \mathrm{~Gy}$ for feasibility of injection, and seven dogs were treated with a single treatment to a BED of $105 \mathrm{~Gy}$. The BED of 105 Gy was used as it is standard dose administered to men with prostate cancer receiving brachytherapy. ${ }^{21,22}$ Ultrasoundguided injection was used for the first dog treated, and due to the less than ideal imaging characteristics of ultrasound for needle placement, CT was used to guide needle placement in the remaining eight dogs. The total injected activity was based on prostate tumor size as outlined in Table 1 . The target injection dose was $0.17 \mathrm{mCi}$ per $\mathrm{cm}^{3}$ assuming $80 \%$ retention as seen in mouse models. Dogs were housed in radiation isolation for a period of 5-9 days, until released by the radiation safety officer. During this time, a standardized quality of life form was completed once daily for six dogs in the BED of 105 Gy group. One dog had no evidence of changes in posture, attitude, or appetite. One dog had one episode of vomiting 7 days posttreatment, but no other evidence of discomfort or gastrointestinal abnormalities. Three dogs had a transient decrease in appetite lasting from 1 to 3 days after treatment; appetite recovered without intervention in all three dogs. One dog was restless and had a decreased appetite for 4 days following treatment, but subsequently recovered with no intervention. One dog, that also experienced a transient decrease in appetite, needed to have manual bladder expression twice following injection for bladder emptying prior to resuming normal voluntary urination. No dog required interventional therapy within 7 days posttreatment, and no other acute side effects were clinically evident immediately following treatment.

Local complications were potentially seen in one of the seven dogs in the BED of 105 Gy group. This dog was treated to a BED of $105 \mathrm{~Gy}$ and had a partial urethral obstruction present prior to presentation; urethral stenting was not available at our institution at this time. Twelve days following injection, and 5 days following discharge to dog owners, a full urethral obstruction resulted in bladder rupture and death of this dog. Full postmortem examination was not performed; however, the prostate and bladder were examined. Tumor progression leading to full urethral obstruction versus edema caused by injection of GA- ${ }^{198} \mathrm{AuNPs}$ and resultant inflammation could not be determined.

Imaging was planned in all nine dogs 4 weeks following treatment with GA- ${ }^{198} \mathrm{AuNP}$; eight dogs had a CT scan 4 weeks postinjection; one dog died prior to week 4 as described above. There was no evidence of tumor swelling, abscessation, or cyst formation in any dog imaged. Although changes in tumor volume were not an endpoint of this study, most dogs had stable disease using three-dimensional tumorvolume measurements. Two dogs had a decrease in tumor volume of $30 \%$ and $50 \%$, respectively, and one dog had an increase in tumor volume of $26 \%$. The other six dogs in this study had minimal changes in tumor volume. Representative pre- and posttreatment images in one of the treated dogs with $50 \%$ tumor-volume reduction is shown in Figure 1; these images also reveal no evidence of tumor edema or abscess formation following treatment. Pre- and posttreatment tumor volumes are detailed in Table 1.

Systemic toxicity in treated dogs was evaluated by measuring blood parameters prior to treatment and then weekly for 4 weeks. Six of nine dogs completed the weekly bloodwork for the 4-week trial period, all in the 105 Gy BED group; two dogs in the BED 50 Gy group had only pretreatment and 4-week posttreatment bloodwork performed. One dog died prior to completion of study, having only pretreatment and 1-week posttreatment parameters measured. The complete blood count and serum chemistry parameters are provided in Table 2 . There were no statistically significant differences between pre- and posttreatment values for the following parameters for dogs in the 105 Gy BED group: total white blood cell count $(P=0.8203)$; total red blood cell count ( $P=0.3594)$; neutrophil count $(P=0.5703)$; platelet count $(P=1.0000)$; alkaline phosphatase $(P=0.2422)$; alanine aminotransferase $(P=0.7422)$; blood urea nitrogen $(P=1.0000)$; and creatinine $(P=1.0000)$. Mean weight at study enrollment was $24.5 \mathrm{~kg}$ (range 6.6-39.8 kg) and $23.5 \mathrm{~kg}$ (range 6.7-39 kg) at study completion.

Whole body planar imaging was performed within 30 minutes postinjection in seven dogs in the 105 Gy BED group using a window centered on the $412 \mathrm{keV}$ photon peak and a $+/-$ window of $10 \%$ to monitor distribution of radioactivity. The scintigraphic images indicate no evidence of nanoconstruct in the liver and spleen, which are the main target organs for systemically distributed NPs of this type. ${ }^{10,13}$ The planar images obtained within 30 minutes postinjection for seven dogs are shown in Figure 2. Leakage of ${ }^{198} \mathrm{AuNPs}$ into the bladder, urethra, and extraprostatic region was observed in six of the seven dogs and is indicated in Figure 2 by yellow (bladder), red (urethra), and white (extraprostatic region) arrows. The localization of GA- ${ }^{198}$ AuNP was calculated for each dog using ROI evaluation and ranged from $21 \%$ to $90 \%$ with an average of $53 \%$. The main source of radioactivity loss was through the urinary tract via direct drainage of NPs into the urethra (four dogs) and bladder (three dogs). 

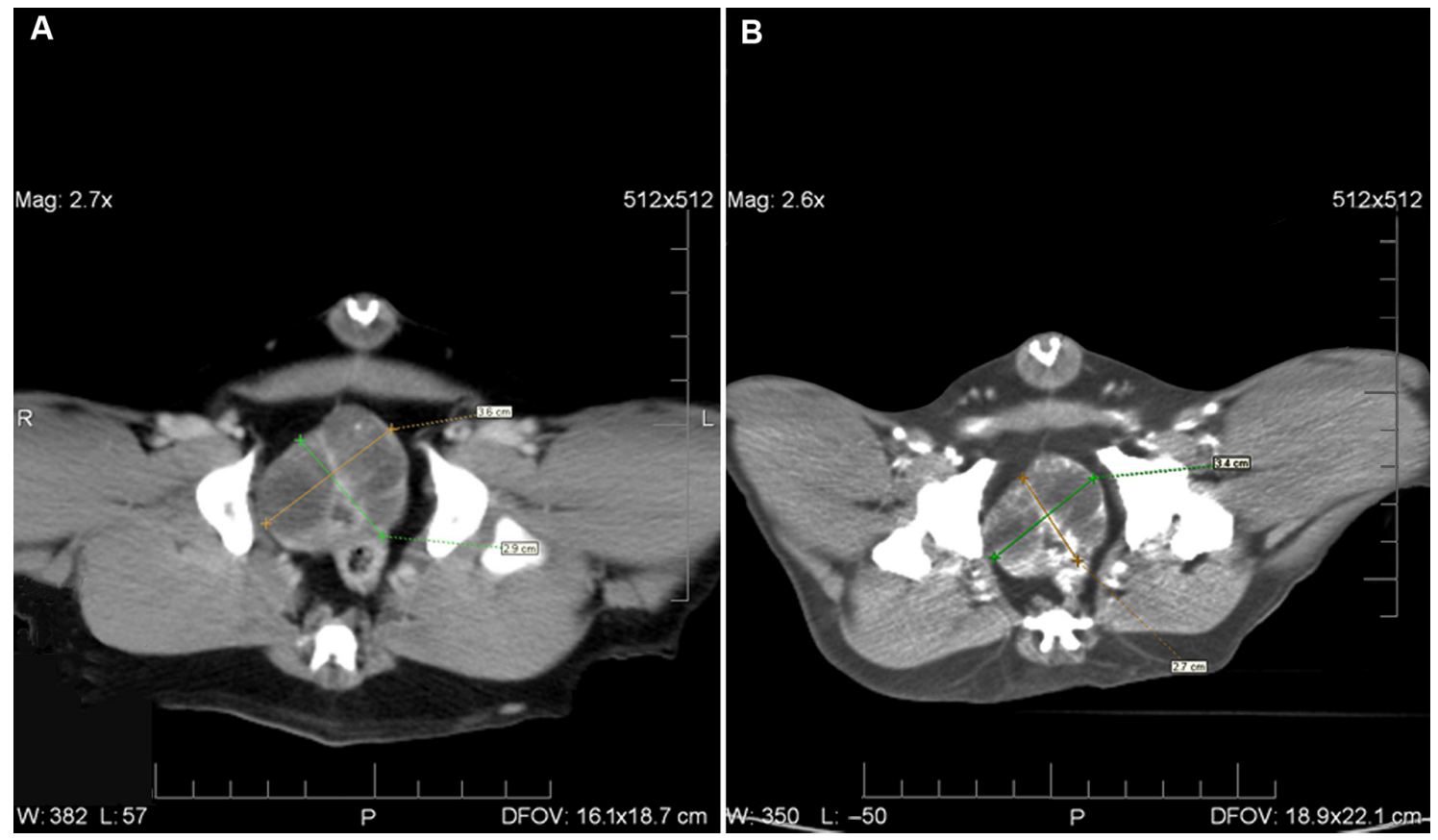

Figure I Pre- and 4-week postinjection CT images of one of the dogs with prostatic carcinoma treated with GA- ${ }^{198} \mathrm{AuNP}$ that showed $50 \%$ tumor volume reduction. Notes: (A) CT image of prostate carcinoma of the dog prior to treatment; (B) posttreatment CT image of same dog's prostate tumor after 4 weeks. These images were chosen to represent the cross-section of the prostate at the same level; respiration, changes in bowel position, size and position of the urinary bladder, and the angle at which the pelvis is positioned affect the position of the prostate within the pelvic cavity between the two imaging studies. Scale bars representing I $\mathrm{cm}$ per division on these images are at the right hand side of the image. Prostatic volume was calculated in two different ways: the standard geometric formula for calculating the volume of an ellipsoid; and the length $\times$ width $\times$ height measurements times pi divided by 6 method commonly used in oncology. Measurements were also obtained from a three-dimensional computerized system which calculates the volume of the prostate based on the cross-sectional area and voxel thickness for each section of the prostate.

Abbreviations: $\mathrm{CT}$, computed tomography; GA- ${ }^{198} \mathrm{AuNP}$, gum arabic-coated radioactive gold nanoparticles.

\section{Discussion}

Overall, intralesional injection of GA- ${ }^{198}$ AuNPs was found to be safe in this group of dogs with naturally occurring prostatic cancer. Evaluation of feasibility and safety in this population of dogs with naturally occurring prostate tumors recapitulates the previously demonstrated murine model safety. ${ }^{16}$ Behavioral changes immediately postinjection were minimal and resolved without intervention, and no difference in parameters was found in hematologic or biochemical parameters pre- and posttreatment. Imaging 4 weeks following treatment revealed no evidence of tumor abscessation or cyst formation in any dog imaged. One dog died due to either tumor progression or tumor swelling resulting in complete urethral obstruction; this dog had evidence of partial urethral obstruction due to the prostatic tumor prior to treatment; therefore, if urethral obstruction is present, relief of obstruction via urethral stenting is recommended in the dog model.

Accurate dosimetry is important in any brachytherapy procedure; the same is true for intralesional GA- ${ }^{198} \mathrm{AuNPs}$. Both the homogenous distribution model and the point source model may be accurate with intralesional NPs. Although distribution of gadolinium and ethanol injected into the

Table 2 Complete blood count and serum biochemical panel results in six dogs

\begin{tabular}{|c|c|c|c|c|c|}
\hline Parameter & Pretreatment & Week I & Week 2 & Week 3 & Week 4 \\
\hline Total white blood cells/ $\mu \mathrm{L}$ & $10,228(6,370-16,400)$ & $9,173(6,240-11,210)$ & $8,295(6,080-9,780)$ & $8,456(5,400-17,000)$ & $8,578(5,900-10,740)$ \\
\hline Total red blood cells $\times 10^{6} / \mu \mathrm{L}$ & $6.18(5.6-6.96)$ & $6.55(5.35-7.15)$ & $6.36(4.84-7.08)$ & $6.32(5.34-7.18)$ & $6.33(5.61-6.91)$ \\
\hline Neutrophils/ $\mu \mathrm{L}$ & $8,284(4,008-13,776)$ & $6,468(3,240-9,190)$ & $7,077(4,090-12,920)$ & $4,95 \mid(4,077-12,920)$ & $6,365(4,250-7,980)$ \\
\hline Platelets $\times 10^{3} / \mu \mathrm{L}$ & $36 \mid(217-488)$ & $478(275-57 I)$ & $42 I(266-53 I)$ & $425(338-548)$ & $334(131-534)$ \\
\hline Alkaline phosphatase U/L & $150(39-433)$ & $168(28-454)$ & $|2|(25-3 \mid I)$ & $88(32-293)$ & $123(26-265)$ \\
\hline Alanine aminotransferase U/L & $32(17-49)$ & $31(16-48)$ & $34(17-73)$ & $39(|0-7|)$ & $35(23-60)$ \\
\hline Blood urea nitrogen $\mathrm{mg} / \mathrm{dL}$ & $20(14-33)$ & I8 (I3-29) & $23(10-29)$ & $22(13-36)$ & $20(16-29)$ \\
\hline Creatinine $\mathrm{mg} / \mathrm{dL}$ & $0.9(0.6-1)$ & $0.95(0.8-1.4)$ & $1.02(0.6-1.7)$ & $1.03(0.6-1.6)$ & $0.96(0.6-1.4)$ \\
\hline
\end{tabular}

Note: Data are represented as the mean and range. 

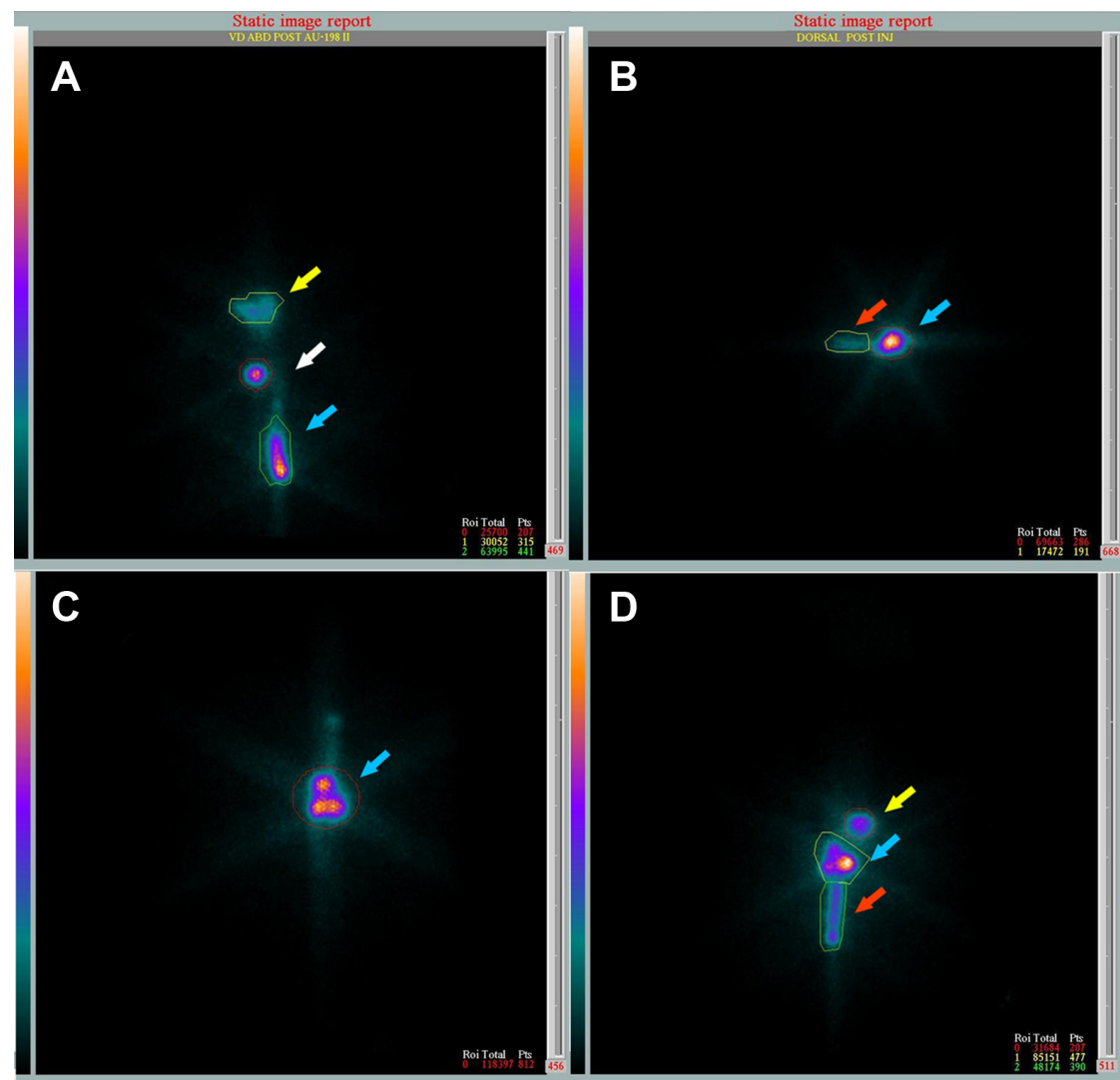

D
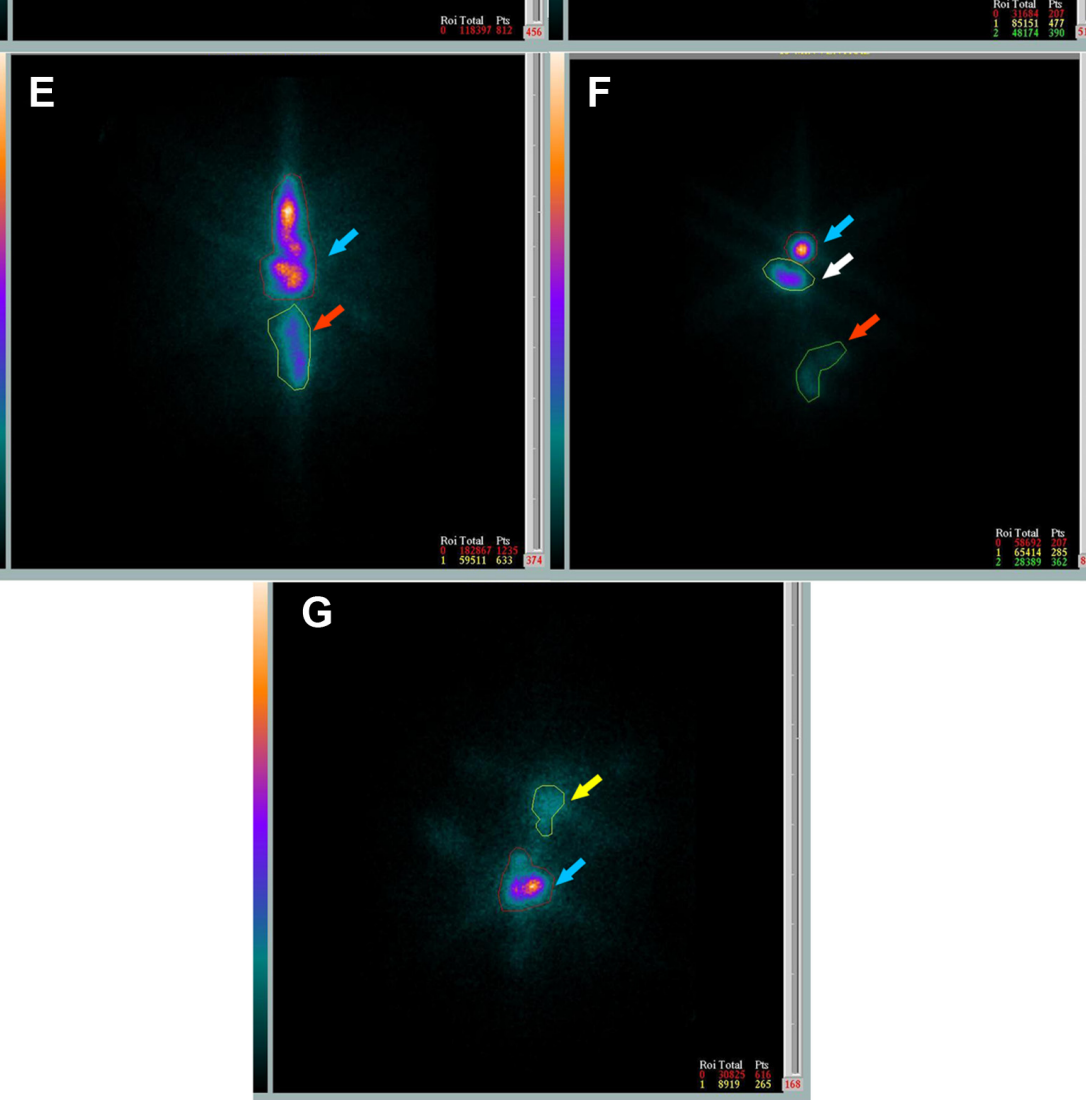

Figure 2 Scintigraphic images recorded within 30 minutes postinjection of GA- ${ }^{198} \mathrm{AuNP}$ in seven dogs.

Notes: (A-G) Dogs three to nine, respectively. These images reveal presence of radioactive gold nanoparticles within the prostate postinjection as indicated by the blue arrows; the multiple injection points within the prostate tumor are clearly seen in one of the dogs (C). Leakage of small amounts of radioactive gold nanoparticles into bladder, urethra, and extra prostate region following injection in six of seven dogs is noted by the yellow, red, and white arrows, respectively. Abbreviation: GA- ${ }^{198} \mathrm{AuNP}$, gum arabic-coated radioactive gold nanoparticles. 
prostate have been studied in normal dogs, these models do not take into account NPs size, porous tumor vasculature, poor tumor lymphatic drainage, or tumor tissue structure, all of which are integral to determining distribution and retention of GA- ${ }^{198}$ AuNP. ${ }^{23}$ Additionally, in dogs, PC obliterates normal prostate gland structure and in most dogs normal prostate is not detectable. ${ }^{24}$ Therefore, in the present study, the homogenous distribution model was used for calculation of the delivered dose as this was the dose distribution observed in the murine implanted tumor model. ${ }^{13,15,16}$ In this model, virtually the entire dose deposition is assumed to be from beta particle emissions of ${ }^{198} \mathrm{Au}$ 's decay; these particles have a range of $1.0 \mathrm{~mm}$ in tissue with a homogenous backscatter pattern. Because of its high energy, the $412 \mathrm{keV}$ gamma ray has less than 1\% local absorption and is therefore overlooked in the dosimetry calculation. Even though it is unlikely that diffusion of GA- ${ }^{198}$ AuNPs is completely uniform due to the presence of tissue planes, blood vessels, and vestigial structures, during injection, interstitial pressure is generated that is great enough for migration of the suspension along paths of least resistance. Based on the planar images in Figure 2, it is evident that diffusion is taking place by partial loss of agent from the prostate into the urethra and bladder. Therefore, the homogenous distribution model is the more appropriate of the two dosimetry models for this agent. Prior to studies in men, injections administered into human prostates ex vivo may be helpful in dosimetry, as normal tissue structure played a role in gadolinium distribution and leakage, and residual normal prostate tissue is present in many men with prostate cancer. ${ }^{23}$ Further study is ongoing to confirm the ideal dosimetric scheme in dogs; once these studies are complete, further studies will be performed to both confirm safety and study efficacy in this model prior to Phase I trials in men.

Many methods are available to detect gold NPs in tissues. CT, micro-CT, MRI, and optical radiance have been described in various models. ${ }^{25-29}$ In this study, we elected to use nuclear scintigraphy to detect radioactivity associated with the gold NPs. Images at later time points should be considered in future studies to monitor the distribution of radioactivity within the prostate tumors in addition to $\mathrm{CT}$ scans to better predict dispersal of GA- ${ }^{198} \mathrm{AuNPs}$ following injection.

A BED of 105 Gy was calculated for seven dogs in the treatment arm of this study, and was adjusted for $>75 \%$ mean tumor retention seen in mouse models. ${ }^{16}$ Planar images recorded in seven dogs within 30 minutes postinjection confirmed the presence of GA- ${ }^{198}$ AuNP within the prostate in all seven dogs. As prostatic tumors in dogs obliterate normal prostate structure, no to minimal delineation between normal prostate and tumor tissue was visualized on CT scan in this group of dogs; therefore, we assumed that the total amount of GA- ${ }^{198} \mathrm{AuNP}$ estimated in prostate through ROI evaluation by planar imaging is expected to be present in the tumor. The average amount of GA- ${ }^{198} \mathrm{AuNP}$ localized in prostate at 30 minutes postinjection based on planar images is $\sim 53 \%$ and is lower than that seen in mice models $(154.05 \% \pm 40.7 \%$ injected dose/g) at 30 minutes. ${ }^{16}$ The reduced tumor retention is likely more reflective of what might be expected in men with naturally occurring prostate cancer and underscores the importance of using spontaneously occurring disease in dogs as models to bridge the gap between murine models and people. Given the erratic nature of tumor vasculature and poor lymphatic drainage in most solid tumors, ${ }^{11}$ nuclear scintigraphy at later time points would be necessary to determine long-term retention, distribution, and diffusion of GA- ${ }^{198} \mathrm{AuNP}$. Prior to Phase I studies in men, further investigation to optimize dosimetry is indicated. Injection into the tumor was guided by ultrasound or CT imaging, and the invasive nature of these tumors dictates that some ductal structures are likely to remain within grossly affected tumor tissue and be responsible for loss of agent through the bladder.

Toxicity of gold in tissues is an important consideration in gold NP treatment. Similar to previous studies using inert gold NPs, toxicities to normal tissues was minimal. ${ }^{30}$ Toxicity was measured systemically with complete blood counts for bone marrow function, serum biochemical profile, and urinalysis for hepatic and renal function, and locally with CT scan 4 weeks posttreatment. Quality of life forms were used during the initial hospitalization period. Dogs showed minimal behavioral signs of discomfort or systemic malaise. There was no change in white blood cell, red blood cell, or platelet parameters between weeks demonstrating a lack of bone marrow toxicity in this treated group of dogs. In previous studies, reticuloendothelial system uptake of nonradioactive gold NPs in general and GA-AuNP specifically have been observed upon systemic administration; ${ }^{14,16,30}$ therefore, systemic uptake and toxicity issues related to those were of particular concern in this study. However, no changes in renal parameters or liver enzymes were found between weeks, suggesting no toxicity to these organs. Restlessness and decreased appetite were the most common changes seen following treatment, which could be due to discomfort or in-hospital boarding. These changes resolved spontaneously. One dog in this study died due to urethral obstruction and bladder rupture - due to the unavailability of a full postmortem examination, we were unable to determine 
if this obstruction was caused by tumor progression or delayed inflammation, or infection caused by injection. As most dogs are diagnosed late in the stage of disease, partial or complete urethral obstruction is a common clinical finding, and placement of a urethral stent was recommended in dogs that had difficulty urinating and evidence of obstruction prior to GA- ${ }^{198} \mathrm{AuNP}$ injection when this intervention became available at our institution.

Many studies have been performed investigating the toxicity and distribution of nanoparticulate gold, and both the size and the coating used for gold NPs will significantly affect the distribution and toxicity. ${ }^{31-33}$ For example, a polyethylene glycol coating on a gold NP with a hydrodynamic size of $27.6 \mathrm{~nm}$ in size at doses ranging from $0.17-4.26 \mathrm{mg} / \mathrm{kg}$ results in acute inflammation and apoptosis of hepatocytes following intravenous administration compared to no abnormalities or acute toxicities following intravenous administration of $2 \mathrm{mg} / \mathrm{kg}$ of gum arabic-coated gold NPs with a hydrodynamic size of $85 \mathrm{~nm} .{ }^{31,33}$ In mouse model studies using gold nanoparticles, the median lethal dose is $3.2 \mathrm{~g}$ of Au per kg. ${ }^{34}$ Since dogs in our study were administered much lower doses of gold - a maximum of $0.325 \mathrm{mg}$ of gold - no tissue toxicity from gold alone was anticipated. Toxicity to local tissues caused by ${ }^{198} \mathrm{AuNPs}$ was thought to be a result of cellular uptake and tissue necrosis. Indeed, necrosis was the major finding on histopathologic exam of prostatic carcinoma in tumor-bearing mice treated with intralesional GA- ${ }^{198}$ AuNP. ${ }^{16}$ CT scan 4 weeks following treatment did not reveal evidence of abscess formation or tumor edema. While none of the tests performed to evaluate toxicity are as sensitive as postmortem examination and histopathology, this is an inherent limitation when performing a clinical trial using client-owned animals.

Dogs used in this study were typical of those that develop spontaneously-occurring cancer of the prostate. All dogs in this treatment group were castrated years prior to diagnosis, a finding consistent with a large epidemiological study identifying castration as a risk factor for development of prostate cancer in dogs. ${ }^{35}$ They were typically older dogs and the range of their body size and tumor volume demonstrates the feasibility of intraprostate injection, as all were injected successfully with retention in the target tissue. Similarly, the geometry of their intrapelvic organs is quite similar to men and revealed no early off-target effects of the radiation. Radiation doses administered were much more similar to those that would be prescribed for men than those that are prescribed in mice. Thus, injection volumes are similar and support further feasibility of translation to men. Although efficacy was not an endpoint in this study, it is of particular note that tumor volume stability and even tumor reduction was noted in some dogs. Two of the eight dogs with posttreatment imaging had substantial tumor volume reduction $(30 \%$ and $55 \%$, respectively) and four dogs had relatively stable disease. Prostatic carcinoma in dogs is a rapidly progressive cancer, and stable disease in the face of no other cancer therapy may indicate early efficacy of this treatment. In fact, the 30-day study time period was chosen based on historical data reporting a median survival time of 0-30 days in dogs with untreated prostate cancer. ${ }^{36}$ Further dose-escalation study with a larger number of dogs is warranted to confirm the effectiveness of this therapy in high grade castration-resistant prostate cancer prior to Phase I trials in men.

This study is unique in that it utilizes a naturally occurring model of aggressive prostatic cancer to bridge the gap in translational science between rodent models and humans. Currently, there is no commonly used model of spontaneously occurring PC, and the treatment results obtained in mice with induced PC may not be analogous to expected results in men, nor can local or systemic toxicities be predicted accurately using a rodent model with implanted cancer. Prostate cancer in dogs is high grade and independent of hormonal influence. Similarly, a subset of men develop high grade PC, which becomes independent of hormonal influences late in the disease course and is refractory to most monotherapies. ${ }^{4-9,24}$ Dogs represent an ideal model for PC due to many similarities, including the influence of aging on cancer formation, an intact immune system, and shared environment with men. Additional advantages to the canine model include their use as a large animal model for imaging and treatment within a naturally condensed lifespan. ${ }^{24}$

\section{Conclusion}

Although traditional brachytherapy using ${ }^{103} \mathrm{Pd}$ or ${ }^{125} \mathrm{I}$ seeds are successful in treating men with low grade prostatic cancer, significant numbers of men with intermediate and high grade disease relapse following treatment..$^{2,4,6-9}$ Highlighting the importance of successful irradiation, evidence in the literature suggests that achieving early local control in intermediate and high Gleason score prostatic carcinoma can decrease the risk of disease metastasis. ${ }^{21,22}$ Therefore, treatment options that provide excellent control of local disease while sparing normal tissues can improve both the quality and quantity of life in men with PC. It is hypothesized that due to size, GA- ${ }^{198}$ AuNPs have more homogenous dose distribution and higher emission of energy compared to current brachytherapy seeds used to treat PC. The gum arabic glycoprotein provides 
a nontoxic coating on NPs with a hydrodynamic diameter of $85 \mathrm{~nm}$ and is highly stable in vivo. ${ }^{16}$ The ease of production combined with the properties of ${ }^{198} \mathrm{Au}$ and the safety of GA- ${ }^{198}$ AuNP make this new treatment an exciting advancement in prostatic cancer therapy. While this study was not powered to evaluate outcome, ongoing studies will evaluate long-term efficacy and optimize dosimetry.

This study provides evidence that intralesional injection of GA- ${ }^{198}$ AuNP is safe with minimal short-term systemic toxicity in the naturally occurring large animal model of prostatic cancer. The favorable safety profile of GA- ${ }^{198} \mathrm{AuNP}$ in this group of dogs suggests that further study for dosimetry and therapeutic efficacy in dogs with prostate cancer and subsequent Phase I clinical trials in men are warranted.

\section{Acknowledgments}

Grant and financial support: This work was supported by a grant from the University of Missouri Institute of Clinical and Translational Sciences, NIH-SBIR Phase II contract (HHSN261201000100C), and Shasun NBI, LLC.

\section{Disclosure}

AU, KVK and RK are co-founders of Nanoparticle Biochem, Inc.. The other authors have no conflicts of interest in this work.

\section{References}

1. Siegel R, Naishadham D, Jemal A. Cancer statistics, 2012. CA Cancer J Clin. 2012;62(1):10-29.

2. Crook J. The role of brachytherapy in the definitive management of prostate cancer. Cancer Radiother. 2011;15(3):230-237.

3. Morton GC. The emerging role of high-dose-rate brachytherapy for prostate cancer. Clin Oncol (R Coll Radiol). 2005;17(4):219-227.

4. Dauffy LS, Braby LA, Berner BM. Dosimetry of the 198Au source used in interstitial brachytherapy. Med Phys. 2005;32(6):1579-1588.

5. Podder TK, Dicker AP, Hutapea P, Darvish K, Yu Y. A novel curvilinear approach for prostate seed implantation. Med Phys. 2012;39(4): $1887-1892$.

6. Stone NN, Stone MM, Rosenstein BS, Unger P, Stock RG. Influence of pretreatment and treatment factors on intermediate to long-term outcome after prostate brachytherapy. J Urol. 2011;185(2):495-500.

7. Stone NN, Stock RG. Prostate brachytherapy: treatment strategies. J Urol. 1999;162(2):421-426.

8. Butler EB, Scardino PT, Teh BS, et al. The Baylor College of Medicine experience with gold seed implantation. Semin Surg Oncol. 1997;13(6): 406-418.

9. Potters L, Klein EA, Kattan MW, et al. Monotherapy for stage T1-T2 prostate cancer: radical prostatectomy, external beam radiotherapy, or permanent seed implantation. Radiother Oncol. 2004;71(1): 29-33.

10. Kannan R, Rahing V, Cutler C, et al. Nanocompatible chemistry toward fabrication of target-specific gold nanoparticles. $\mathrm{J} \mathrm{Am} \mathrm{Chem}$ Soc. 2006;128(35):11342-11343.

11. Waite CL, Roth CM. Nanoscale drug delivery systems for enhanced drug penetration into solid tumors: current progress and opportunities. Crit Rev Biomed Eng. 2012;40(1):21-41.
12. Papasani MR, Wang G, Hill RA. Gold nanoparticles: the importance of physiological principles to devise strategies for targeted drug delivery. Nanomedicine. 2012;8(6):804-814.

13. Kattumuri V, Katti K, Bhaskaran S, et al. Gum arabic as a phytochemical construct for the stabilization of gold nanoparticles: in vivo pharmacokinetics and X-ray-contrast-imaging studies. Small. 2007;3(2): 333-341.

14. Boote E, Fent G, Kattumuri V, et al. Gold nanoparticle contrast in a phantom and juvenile swine: models for molecular imaging of human organs using x-ray computed tomography. Acad Radiol. 2010;17(4): 410-417.

15. Kannan R, Zambre A, Chanda N, et al. Functionalized radioactive gold nanoparticles in tumor therapy. Wiley Interdiscip Rev Nanomed Nanobiotechnol. 2012;4(1):42-51.

16. Chanda N, Kan P, Watkinson LD, et al. Radioactive gold nanoparticles in cancer therapy: therapeutic efficacy studies of GA-198AuNP nanoconstruct in prostate tumor-bearing mice. Nanomedicine. 2010;6(2): 201-209.

17. Li Y, Wu P, Xu H, Zhang H, Zhong X. Anti-aggregation of gold nanoparticle-based colorimetric sensor for glutathione with excellent selectivity and sensitivity. Analyst. 2011;136(1):196-200.

18. Whelove OE, Cozad MJ, Lee BD, et al. Development and in vitro studies of a polyethylene terephthalate-gold nanoparticle scaffold for improved biocompatibility. J Biomed Mater Res B Appl Biomater. 2011;99(1): 142-149.

19. Lystvet SM, Volden S, Yasuda M, Halskau Ø Jr, Glomm WR. Emergent membrane-affecting properties of BSA-gold nanoparticle constructs. Nanoscale. 2011;3(4):1788-1797.

20. Rivard MJ, Coursey BM, DeWerd LA, et al. Update of AAPM Task Group No. 43 Report: A revised AAPM protocol for brachytherapy dose calculations. Med Phys. 2004;31(3):633-674.

21. Porter AT, Forman JD. Prostate brachytherapy. An overview. Cancer. 1993;71(3 Suppl):953-958.

22. Wilt TJ, MacDonald R, Rutks I, Shamliyan TA, Taylor BC, Kane RL. Systematic review: comparative effectiveness and harms of treatments for clinically localized prostate cancer. Ann Intern Med. 2008;148(6): 435-448.

23. Chowning SL, Susil RC, Krieger A, Fichtinger G, Whitcomb LL, Atalar E. A preliminary analysis and model of prostate injection distributions. Prostate. 2006;66(4):344-357.

24. Leroy BE, Northrup N. Prostate cancer in dogs: comparative and clinical aspects. Vet J. 2009;180(2):149-162.

25. Liang $\mathrm{CH}$, Wang $\mathrm{CC}$, Lin $\mathrm{YC}$, Chen $\mathrm{CH}$, Wong $\mathrm{CH}, \mathrm{Wu} \mathrm{CY}$. Iron oxide/gold core/shell nanoparticles for ultrasensitive detection of carbohydrate-protein interactions. Anal Chem. 2009;81(18):7750-7756.

26. Conversano F, Soloperto G, Greco A, et al. Echographic detectability of optoacoustic signals from low-concentration PEG-coated gold nanorods. Int J Nanomedicine. 2012;7:4373-4389.

27. Hainfeld JF, O'Connor MJ, Dilmanian FA, Slatkin DN, Adams DJ, Smilowitz HM. Micro-CT enables microlocalisation and quantification of Her2-targeted gold nanoparticles within tumour regions. Br J Radiol. 2011;84(1002):526-533.

28. Reuveni T, Motiei M, Romman Z, Popovtzer A, Popovtzer R. Targeted gold nanoparticles enable molecular CT imaging of cancer: an in vivo study. Int J Nanomedicine. 2011;6:2859-2864.

29. Grabtchak S, Tonkopi E, Whelan WM. Optical detection of gold nanoparticles in a prostate-shaped porcine phantom. J Biomed Opt. 2013; 18(7):077005.

30. Johnston HJ, Hutchison G, Christensen FM, Peters S, Hankin S, Stone $\mathrm{V}$. A review of the in vivo and in vitro toxicity of silver and gold particulates: particle attributes and biological mechanisms responsible for the observed toxicity. Crit Rev Toxicol. 2010;40(4):328-346.

31. Li YF, Chen C. Fate and toxicity of metallic and metal-containing nanoparticles for biomedical applications. Small. 2011;7(21):2965-2980.

32. De Jong WH, Hagens WI, Krystek P, Burger MC, Sips AJ, Geertsma RE. Particle size-dependent organ distribution of gold nanoparticles after intravenous administration. Biomaterials. 2008;29(12):1912-1919. 
33. Fent GM, Casteel SW, Kim DY, et al. Biodistribution of maltose and gum arabic hybrid gold nanoparticles after intravenous injection in juvenile swine. Nanomedicine. 2009;5(2):128-135.

34. Hainfeld JF, Slatkin DN, Focella TM, Smilowitz HM. Gold nanoparticles: a new X-ray contrast agent. Br J Radiol. 2006;79(939): 248-253.
35. Bryan JN, Keeler MR, Henry CJ, Bryan ME, Hahn AW, Caldwell CW. A population study of neutering status as a risk factor for canine prostate cancer. Prostate. 2007;67(11):1174-1181.

36. Cornell KK, Bostwick DG, Cooley DM, et al. Clinical and pathologic aspects of spontaneous canine prostate carcinoma: a retrospective analysis of 76 cases. Prostate. 2000;45(2):173-183.

\section{Publish your work in this journal}

The International Journal of Nanomedicine is an international, peerreviewed journal focusing on the application of nanotechnology in diagnostics, therapeutics, and drug delivery systems throughout the biomedical field. This journal is indexed on PubMed Central, MedLine, CAS, SciSearch $®$, Current Contents $\circledR /$ Clinical Medicine,
Journal Citation Reports/Science Edition, EMBase, Scopus and the Elsevier Bibliographic databases. The manuscript management system is completely online and includes a very quick and fair peer-review system, which is all easy to use. Visit http://www.dovepress.com/ testimonials.php to read real quotes from published authors.

Submit your manuscript here: http://www.dovepress.com/international-journal-of-nanomedicine-journal 